\title{
Symmetry and asymmetry from MEMS variable capacitor by nonlinear micro stoppers
}

\author{
Jianxiong Zhu ${ }^{1, *}$ (D), Yunde $\mathrm{Shi}^{1}$, and Feng $\mathrm{Ma}^{2,3}$ \\ ${ }^{1}$ School of Mechanical Engineering, Southeast University, Nanjing 211189, PR China \\ ${ }^{2}$ Hunan Zhongtai Special Equipments Co. LTD, Changde 415000, PR China \\ ${ }^{3}$ College of Chemistry and Materials Engineering, Hunan University of Arts and Science, Changde 415000, PR China
}

Received: 19 March 2021 / Accepted: 21 July 2021

\begin{abstract}
Mechanical stoppers in MEMS capacitive systems can dramatically affect electrical performances and result in complicated mechanical dynamic responses. This paper introduces electromechanical coupling nonlinear dynamic responses in MEMS variable dual-capacitor with an effect of nonlinear and asymmetrical stoppers. We found that the capacitance in the electrical circuit system related to the first-order derivative of the output voltage on a load resistor, and the variable dual-capacitor was strongly affected by the coupling of up and down superposition instantaneous electrostatic force and limited space by the length of nonlinear stoppers. The numerical calculation results and the experimental results in our analysis based on our system had a good agreement, and the numerical simulation results presented rich nonlinear impacts dynamic responses through the imposed voltage and the height of stoppers in MEMS variable dual-capacitive device. The device in operation cannot reach the 0.6 time's initial gap due to small forcing amplitude $(1.026 \mathrm{~g})$. However, we observed that the movable plate and stoppers (across the 0.6 time's initial gap) had fierce impacts due to big forcing amplitude $(4 \mathrm{~g})$ on to the device. With asymmetric stopper each impact, we also concluded that the movable plate would experience attenuations of the displacement until the moment to the next impacts. Moreover, the height of stoppers can not only result in complicated dynamic motion of the movable plate, but also can modulate a voltage of the fixed plate with its asymmetry structure.
\end{abstract}

Keywords: Nonlinear stopper / asymmetry and symmetry / softening effect / MEMS variable capacitor / numerical simulation

\section{Introduction}

Energy harvesting from Micro-Electro-Mechanical Systems (MEMS) capacitive devices is a hot research area around mechanical vibration. The power from MEMS capacitive devices can be obtained to support MEMS electronic devices and the Internet of Things. For the capacitive device in operation, the parameters of the capacitor would be changed along with the environment. Thus, the research on the variable capacitive device is of engineering interest, especially on the support for low power consumption devices [1-6]. The principle of MEMS variable capacitive device is based on mechanical vibration to make a change of charges which in turn affects the voltage of charged capacitor [3,4]. Compared to the theory of the electromagnetic [7], the piezoelectric [8], the thermoelectric, or the triboelectric mechanism [9-11],

\footnotetext{
* e-mail: mezhujx@seu.edu.cn
}

the capacitive method has the advantage of easy integration with CMOS technology [10-28]. Thus, we have chosen to work with the capacitive device for both CMOS compatible and simple MEMS process fabrication [29,30].

As to the key parameters of the capacitor, many researchers have researched the parallel plate capacitors with nonlinear electrostatic, nonlinear cantilever springs (hanger), and squeezed film damping things [20,21,31-35]. Qiu et al. discussed the softening effect of their capacitive device with a strong nonlinear component in the system [20]. Nayfeh et al. reported superharmonic and subharmonic nonlinear analysis of the system in experiments [21]. They focused on the mechanism of the modeling in motion without considering the advantage of dual-cavity. Zhu et al. introduced strong nonlinear impact force in the capacitor [36,37]. However, they did not conduct the theory and phenomena analysis on the nonlinear stoppers to their asymmetric. Thus, almost no groups have been conducted an electromechanical coupling of stoppers in the variable dual-capacitive device. Even though the previous research 
(a)

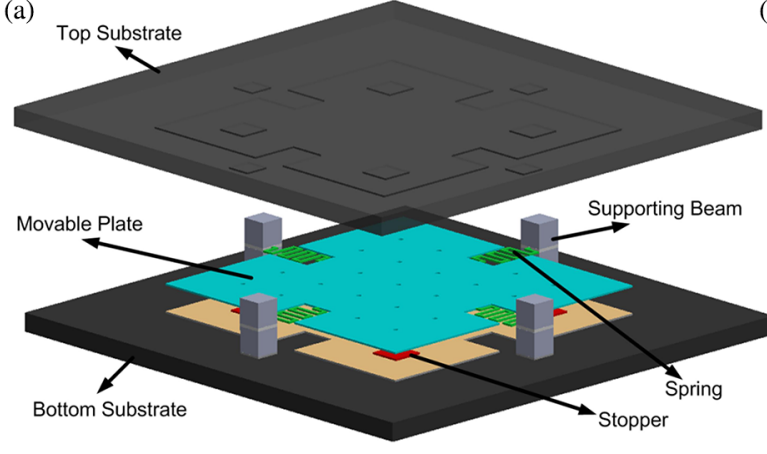

(b)

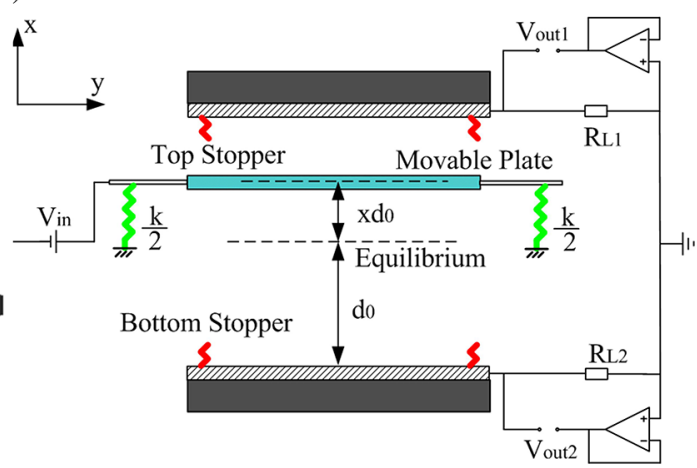

Fig. 1. (a) 3D schematic view of MEMS variable dual-capacitive device, (b) physical model of MEMS variable dual-capacitive device with voltage fluctuation.

showed a kind of energy collecting method with MEMS dual-capacitor, it did not do the numerical analysis in MEMS electromechanical coupling based on stoppers [37].

With an asymmetric stopper in the MEMS system, the motion of the movable plate or beam would experience attenuations of the displacement until the moment to the next impacts or constraints. To solve the issues about the nonlinear stoppers and their asymmetry, in this study, we focused on the electromechanical coupling with nonlinear stoppers for dynamic responses in MEMS variable dual-capacitive devices. We found that the capacitance in the electrical circuit system related to the first-order derivative of the output voltage, and the variable dual-capacitor was strongly affected by the coupling of an electrostatic force and the length of stoppers. The numerical simulation and measured experimental results showed a good agreement with nonlinear dynamic response in MEMS variable dual-capacitive device. Moreover, the height of stoppers can not only result in complicated dynamic motion of the movable plate, but also can modulate a root mean square (RMS) voltage with its asymmetry.

\section{MEMS dual-capacitor and mathematical modeling}

The MEMS variable dual-capacitive device is shown in Figure 1a. It contains a movable plate, two fixed plates, and four gold stoppers. The stoppers were designed to avoid the movable plate having impacts with fixed plates overwhelming electrostatic force. The movable plates were hanged by four suspending springs. The fixed plates are located with the sandwich structure to the movable plates. The gold was made as a conductive material, and the chromium was as medium materials as a paste between gold and $\mathrm{SiO}_{2} / \mathrm{Si}_{3} \mathrm{~N}_{4}$ dielectrics. The energy can be generated by the motion of the movable plate due to the electrostatic effect. Four gold stoppers were placed on both the top and bottom fixed plates to prevent snap-down of the movable plates by overwhelming electrostatic force. Figure 1b illustrates a physical model of MEMS variable dual-capacitive device with a voltage fluctuation under a

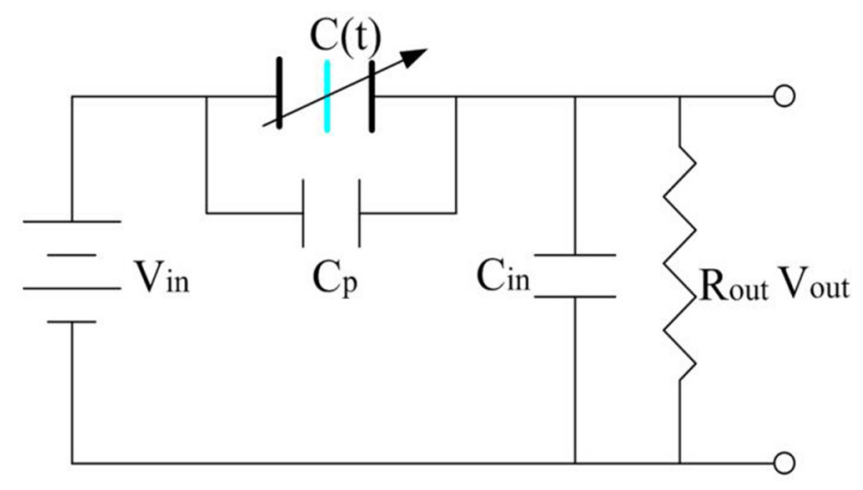

Fig. 2. Electrical circuit of MEMS device. Note that $V_{\text {out }}$ demonstrates $V_{\text {out } 1}$ or $V_{\text {out } 2}$, and $R_{\text {out }}$ is $R_{L 1}$ or $R_{L 2}$.

sinusoidal vibration. An input voltage such as $6 \mathrm{~V}, 9 \mathrm{~V}$, $12 \mathrm{~V}$, or $15 \mathrm{~V}\left(V_{i n}\right)$ was applied to the system with charges initially. The input voltage could be any number. However, most electronic devices are with voltages such as $6 \mathrm{~V}$ or $15 \mathrm{~V}$. To meet the requirement of the electronic devices, we chose the voltage range of $6 \mathrm{~V}-15 \mathrm{~V}$. If the abovementioned voltages were applied onto the device, the movable plate was driven into motion by inertial forces from the charged plates. If with mechanical excitation onto the plates, the voltage over the capacitor increased since the constant input voltage. The load resistors $R_{L 1}$ and $R_{L 2}$, and variable $x$ were used to measure the fluctuation of output voltage during the test and dimensionless displacement of the movable plate from the equilibrium position, respectively.

Figure 2 depicts an electrical circuit of MEMS capacitive device. The ordinary differential equation (ODE) analysis was conducted on an electrical circuit to obtain a relationship between the measured output voltage and the variable capacitance. Parameter $C(t)$ was a variable capacitor. $C_{p}$ and $C_{i n}$ represented a parasitic capacitance and an input capacitance of the voltage measurement circuit which was directly related to the measured output voltage, respectively. The $C_{i n}$ in our case was from the measurement equipment oscilloscope. Since the effect of $C_{p}$ can be lumped into $C(t)$, we used $C_{p}=0$ to describe the electrical circuit [19-21]. The input 
voltages $V_{i n}$ on the top and bottom of the capacitive plates were constant voltage source which was used to provide an initial charge. The bias voltage over $C(t)$ was $V_{\text {in }}-V_{\text {out }}$, which was limited by a pull-in effect and related to the motion of the movable plate. The charge $Q$ would be as follows

$$
Q=C(t)\left(V_{\text {in }}-V_{\text {out }}\right),
$$

the derivative on both sides of the equation was as follows,

$$
\dot{Q}=\dot{C}(t)\left(V_{\text {in }}-V_{\text {out }}\right)-C(t) \dot{V}_{\text {out }} .
$$

The charge over $C_{i n}$ was given by

$$
Q_{1}=C_{\text {in }} V_{\text {out }} .
$$

Based on Kirchhoff current law in the electrical circuit, we had

$$
\dot{Q}=\dot{Q}_{1}+\frac{V_{\text {out }}}{R_{\text {out }}} .
$$

Therefore, we got

$$
\dot{C}(t)\left(V_{\text {in }}-V_{\text {out }}\right)-C(t) \dot{V}_{\text {out }}=C_{\text {in }} \dot{V}_{\text {out }}+\frac{V_{\text {out }}}{R_{\text {out }}} .
$$

By rearranging the terms in equation (5), we finally got

$$
R_{\text {out }}\left[C_{\text {in }}+C(t)\right] \dot{V}_{\text {out }}+\left[R_{\text {out }} \dot{C}(t)+1\right] V_{\text {out }}=V_{\text {in }} R_{\text {out }} \dot{C}(t)
$$

which was

$$
\dot{V}_{\text {out }}=\frac{\dot{C}(t)\left(V_{\text {in }}-V_{\text {out }}\right)-\frac{V_{\text {out }}}{R_{\text {out }}}}{C_{\text {in }}+C(t)} .
$$

The parasitic capacitance $C_{i n}$ had a negligible effect if the capacitive impedance was much larger than $R_{\text {out }}$. Calculating the ratio (IR) of the capacitive to resistive impedances [22,23], we had

$$
I R=\frac{1}{2 \pi f R_{\text {out }} C_{\text {in }}} .
$$

For $R_{\text {out }}=5 \mathrm{M} \Omega, C_{\text {in }}=10 \mathrm{pF}$, and $f=500 \mathrm{~Hz}$, we had

$$
I R=\frac{1}{2 \pi f R_{\text {out }} C_{\text {in }}}=6.4 .
$$

Therefore, the capacitive impedance was a large number for these parameters. It can be assumed that its effect can be ignored. Based on Figure 2b, using Ohm's law on the load resistor, we had

$$
V_{\text {out }}=R_{\text {out }} \dot{Q},
$$

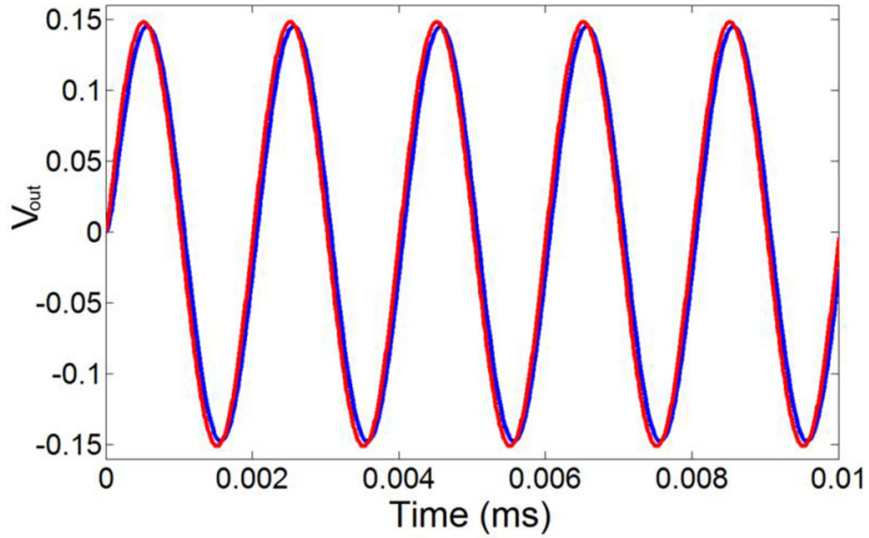

Fig. 3. Calculation results for output voltage.

using capacitance definition and Kirchhoff voltage law on the variable capacitor, we had

$$
Q=C(t)\left(V_{\text {in }}-V_{\text {out }}\right),
$$

by combining equation (10) and equation (11), we obtained

$$
V_{\text {out }}=R_{\text {out }}\left[\left(V_{\text {in }}-V_{\text {out }}\right) \dot{C}(t)-C(t) \dot{V}_{\text {out }}\right]
$$

which is

$$
\dot{V}_{\text {out }}=\frac{\left(V_{\text {in }}-V_{\text {out }}\right) \dot{C}(t)-\frac{V_{\text {out }}}{R_{\text {out }}}}{C(t)} .
$$

Here, $f=500 \mathrm{~Hz}, R_{\text {out }}=5 \mathrm{M} \Omega, V_{\text {in }}=15 \mathrm{~V}, C_{\text {in }}=10 \mathrm{pF}$. With those parameters, $f, R_{\text {out }}, V_{i n}$, and $C_{i n}$, into equations (7) and (13) and solving for them using the first-order ODE in MATLAB. The calculation results from equations (13) and (7) can be found in Figure 3. The curves are in good agreement.

Based on the test results, we found $V_{\text {out }} \ll V_{\text {in }}$. The maximum $V_{\text {out }}$ was less than $0.1 \mathrm{~V}$, and $V_{\text {in }}$ was $15 \mathrm{~V}$. Therefore, we approximated equation (11) by

$$
Q=C(t)\left(V_{\text {in }}-V_{\text {out }}\right) \approx C(t) V_{\text {in }} .
$$

Substituting into equation (10), we can get

$$
V_{\text {out }}=R_{\text {out }} V_{\text {in }} \dot{C}(t)
$$

We found $V_{\text {out }}$ had a harmonic solution based on test results. Thus, we assumed

$$
V_{\text {out }}=A \sin (2 \pi f t),
$$

We can get

$$
C(t)=C_{0}-\frac{A}{2 \pi f R_{\text {out }} V_{\text {in }}} \cos (2 \pi f t)
$$

where $C_{0}=3.54 \mathrm{pF}$, the fluctuation range of $C(t)$ can be determined. Note that the capacitor was directly related to the mechanical motion of the movable plate. 


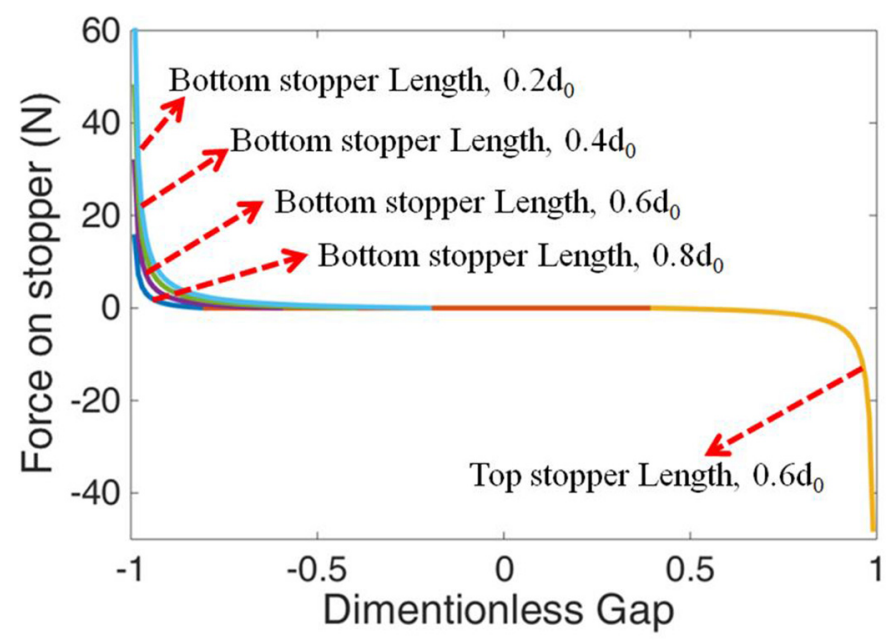

Fig. 4. Nonlinear stoppers with different heights and their asymmetry.

The dynamic responses of the plate and stoppers determine the voltage fluctuation in the MEMS variable dual-capacitive device. Stoppers were not only used to prevent the movable plate and the fixed plates to stick each other but also strongly affected the system's dynamic response by constraining the free range of the movable plate in the gap. A much high constant value of the spring can be assumed as this nonlinear stopper shown in [13-17] as follows

$$
F_{s t}=\left\{\begin{array}{cc}
-k_{s} d_{0} \frac{h+x}{1+x} & -1<x<-h \\
0 & -h<x<s \\
k_{s} d_{0} \frac{s-x}{1-x} & s<x<1
\end{array},\right.
$$

where $s$ and $h$ were the dimensionless stroke parameter from the top stopper and the bottom stopper, respectively. They are normalized by an initial gap $d_{0}$. If parameters $s$ and $h$ with different numbers, it meant that stoppers with an asymmetric state are shown in Figure 4. The stopper spring constant $k_{s}$ was assumed to be 1000 times larger than that of the suspension spring in our case. The capacitance and electrostatic force between two plates can be obtained as follows $[16,24-26]$ :

$$
\begin{gathered}
C(x)=\frac{\varepsilon L W}{d_{0}(1+x)}, \\
F_{e}=-\frac{\partial U}{\partial d}=-\frac{\partial}{\partial d}\left(\frac{Q^{2}}{2 C(x)}\right),
\end{gathered}
$$

where $U$ was potential energy. Based on the theory of superposition of electrostatic forces, the external excitation force on the device can be obtained,

$$
F_{e x}=m \varphi \cos \left(\omega_{e} t\right)
$$

The parameter displacement amplitude $(\varphi)$ in equation (21) was from the d'Alembert force. $\omega e$ was an angle frequency of external vibration. Following Newton's second law and the electrical circuit for MEMS device from equation (13), we had

$$
\begin{gathered}
\ddot{x}=-\frac{c_{1}}{m} \dot{x}-\frac{k}{m} x+\frac{F_{e x}}{m d_{0}}+\frac{F_{\text {st }}}{m d_{0}}+\frac{F_{e}}{m d_{0}}, \\
\ddot{V}_{\text {out }}=\frac{\left(V_{\text {in }}-V_{\text {out }}\right) \dot{C}(t)-\frac{V_{\text {out }}}{R_{\text {out }}}}{C(t)}
\end{gathered}
$$

where $c 1$ was a viscous damping coefficient. Equations (22) and (23) was coupling with each other through a motion of the movable plate. Note that the squeeze film air damping for MEMS device requires a modified Reynolds equation involving the damping effect of gas flow during the plate vibration [27]. The holes in the movable plate were specific designed to reduce the effect of the squeeze film air damping effect. Meanwhile, we assumed that the viscous damping contained the effect of the squeezed film damping. We will perform extensive numerical analysis on the effect of squeeze film air damping in our future work. The power from $R_{\text {out }}$ can be seen as follows

$$
P_{\text {out }}=\frac{V_{\text {out }}^{2}}{R_{\text {out }}} .
$$

\section{Measurement and dynamic response}

The experimental results of MEMS capacitive device were characterized by sweeping excitation frequencies from $200 \mathrm{~Hz}$ to $800 \mathrm{~Hz}$ with steps of $25 \mathrm{~Hz}$ under sinusoidal excitations with constant accelerations, respectively. An RMS output voltage under bias DC of $6 \mathrm{~V}, 9 \mathrm{~V}, 12 \mathrm{~V}$, and $15 \mathrm{~V}$ to each device was plotted as a function of frequency (Fig. 5a). Table 1 shows all the used parameters. The pulldown voltage $(12.4 \mathrm{~V})$ was measured based on equipment to MEMS single capacitor. The goal of the measurement for the pull-down voltage was for the calculation of MEMS spring in the system. It is possible that the MEMS dualcapacitive device still works with $15 \mathrm{~V}$ input voltage. If we applied a higher voltage (18 V) in MEMS dual-capacitive system, the movable plate in the system would stick onto the top of the bottom plate which failed numerical calculation. We found that simulation results matched well with experimental test results (see Fig. 5a,b). The good agreement results verified the feasibility of our analyzed mathematical model. An RMS output voltage meant a voltage perturbation on capacitors as given in equation (11). The voltage perturbation resulted in a small number of nonlinear electrostatic forces on the movable plate. We also found that there was no contact between the movable plate and the fixed plates under $1.026 \mathrm{~g}$ excitation. Compared to $1.026 \mathrm{~g}$ excitations, we found that $4 \mathrm{~g}$ excitations had a much stronger softening effect due to the nonlinear stopper and electrostatic force in the system. The maximum RMS output voltage with $4 \mathrm{~g}$ excitation was 

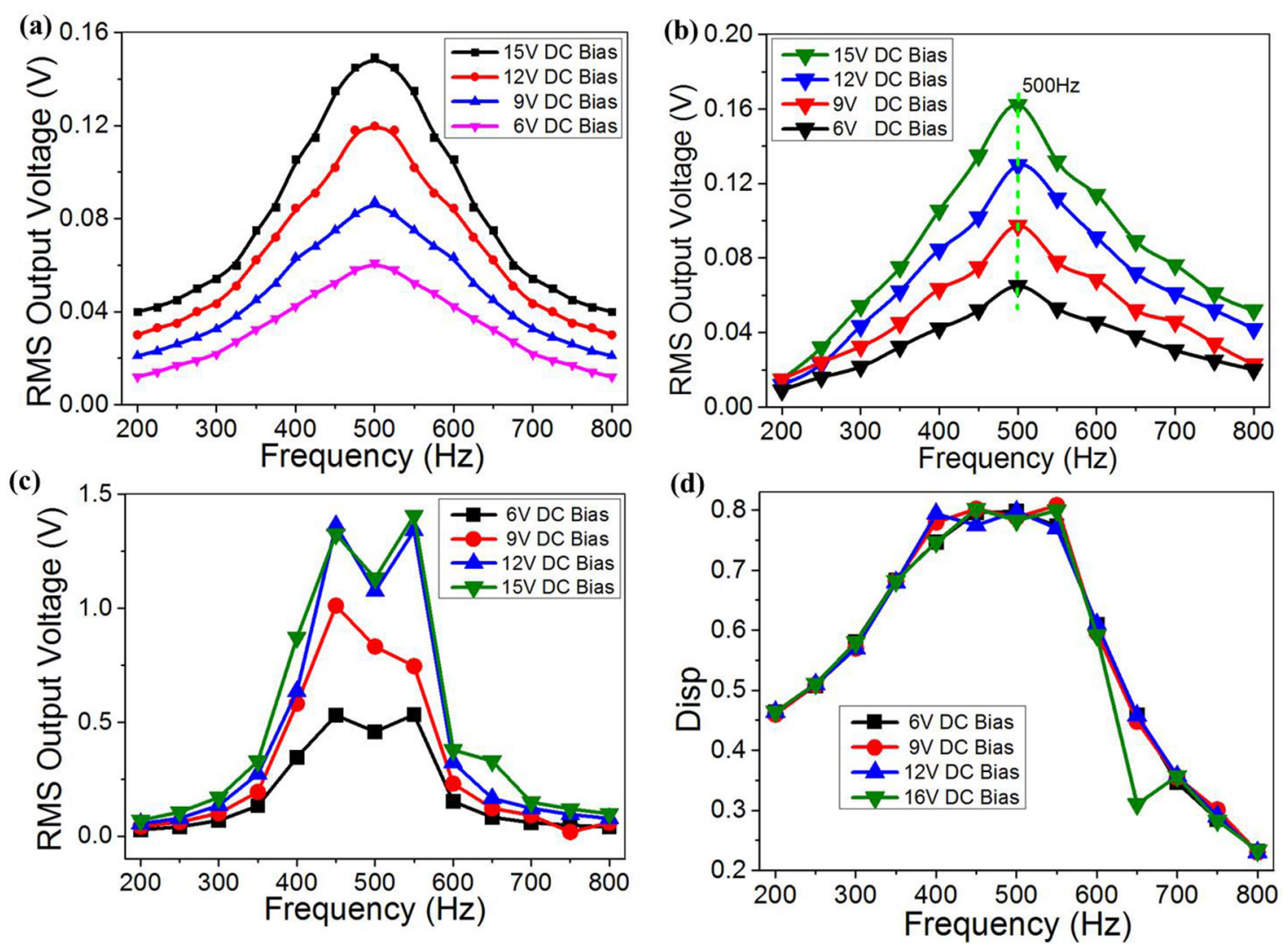

Fig. 5. Measurement: (a) experimental results RMS output voltage with 1.026 g excitation; (b) numerical simulation results in RMS with $1.026 \mathrm{~g}$ excitation; (c) numerical results with $4 \mathrm{~g}$ excitation; (d) numerical simulation results displacement of the movable plate with $4 \mathrm{~g}$ excitation.

Table 1. Simulation parameters.

\begin{tabular}{llll}
\hline System parameters & Symbols & Values & Unit \\
\hline Initial gap & $d_{0}$ & 10 & $\mu \mathrm{m}$ \\
Device resonant frequency & $f_{0}$ & 500 & $\mathrm{~Hz}$ \\
Pull-down voltage & $V_{p d}$ & 12.4 & $\mathrm{~V}$ \\
Movable plate thickness & $h_{1}$ & 30 & $\mu \mathrm{m}$ \\
Movable plate area & $A$ & 25 & $\mathrm{~mm}$ \\
Constant permittivity & $\varepsilon$ & 8.85 & $\mathrm{pF} / \mathrm{m}$ \\
Damping ratio & $\delta$ & 0.2 & 1 \\
Total spring stiffness & $k$ & 168.88 & $\mathrm{~N} / \mathrm{m}$ \\
Stopper spring stiffness & $k_{1}$ & $1.69 \times 10^{5}$ & $\mathrm{~N} / \mathrm{m}$ \\
Displacement limit & $c$ & 0.2 & 1 \\
Height of top stopper & $s$ & 0.6 & 1 \\
\hline
\end{tabular}

constrained in a range of $400-600 \mathrm{~Hz}$ by stoppers (see Fig. 5c, d). It concluded that a higher bias voltage led to more corresponding total output voltage out from MEMS variable dual-capacitor. We can observe the obvious impact between the stoppers and the moving plate from Figure 5d. With an increased amplitude and stopper length $0.6 d 0$, we can get a maximum voltage out of $0.5 \mathrm{~V}$ (see Fig. 6a). We found that the height of stopper 0.2d0 contributed almost 10 times voltage out than $0.8 d 0$ in MEMS variable dual-capacitor (see Fig. 6b). The output voltage $V_{\text {out }}$ followed more or less from equation (15). We concluded that the $V_{\text {out }}$ strongly relied on the limited space with a capacitance derivative of MEMS variable capacitor. The height of stoppers in the system gave an obvious effect for the case with amplitude of $4 \mathrm{~g}$.

The displacements of the movable plate and the output voltage on load resistors are shown in Figure 7. The dynamic response showed that the movable plate did not reach up to stoppers in the dual-capacitive device (see Fig. 7a,b). If MEMS device is subjected to stronger excitation amplitude $(4 \mathrm{~g})$, the dynamic motion of the movable plate changes dramatically due to the impacts between stoppers and movable plate in MEMS dual-cavity device (Eq. (18)). We observed that the impacts between the stoppers and the movable plate increased the energy consumption of the output resistor (see Fig. 7c,d and Eq. (24)).

Figure 8 depicts an investigation of the dynamic response of avoidable asymmetric or symmetric stoppers in MEMS dual-capacitive devices. Figure $8 \mathrm{~b}$ shows that the movable plate impacted with symmetrical stoppers, whereas Figure 8c,d illustrates that the movable plate impacted with asymmetrical stoppers (the bottom stopper $0.4 d 0$ and $0.8 d 0$ ). Compared to symmetric structure, the 

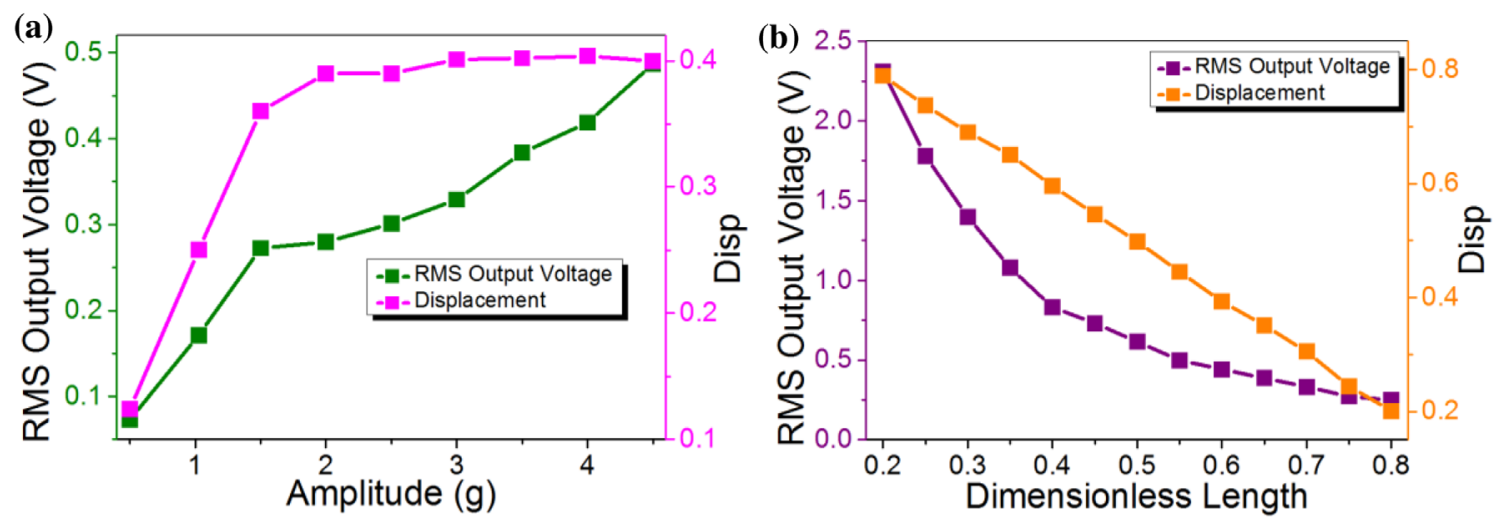

Fig. 6. RMS output voltage and dimensionless displacement of the movable of the plate under external frequency $500 \mathrm{~Hz}, 16 \mathrm{~V}$ input voltage: (a) with different excitation amplitude, stopper length $0.6 d_{0}$; (b) with different length of the stopper, amplitude 4 g.

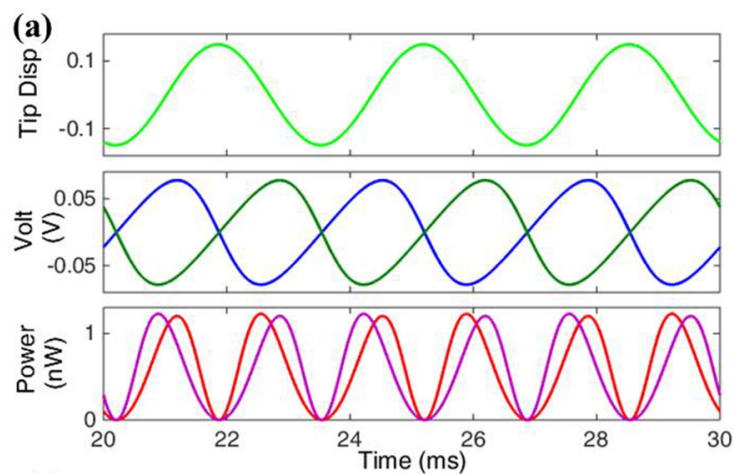

(c)
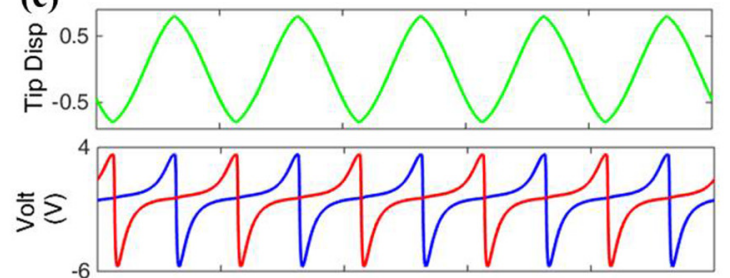

(b)
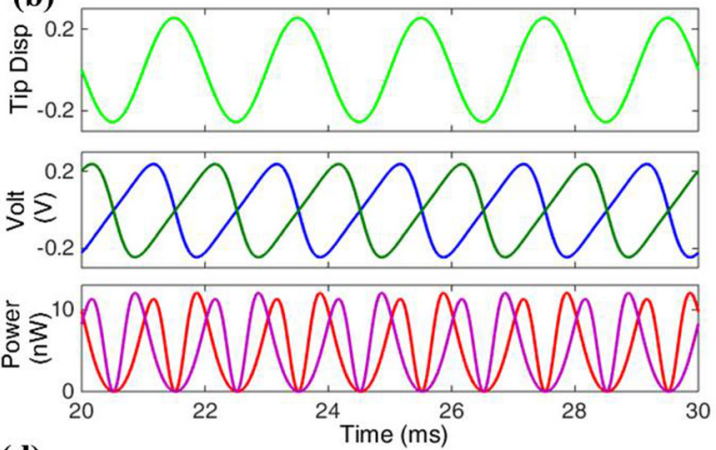

(d)
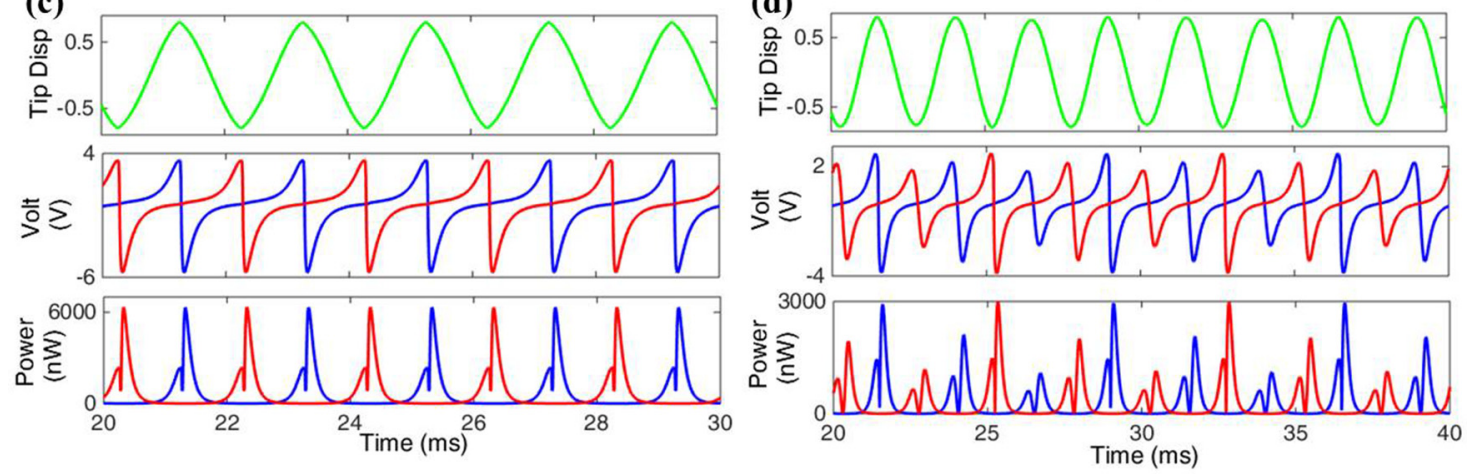

Fig. 7. Numerical results: (a) $400 \mathrm{~Hz}, 1.026 \mathrm{~g}$; (b) $500 \mathrm{~Hz}, 1.026 \mathrm{~g}$; (c) $500 \mathrm{~Hz}, 4 \mathrm{~g}, 2$ impacts in one forcing cycle; (d) $400 \mathrm{~Hz}, 4 \mathrm{~g}, 1.5$ impacts in one forcing cycle.

asymmetric stoppers resulted in much more complicated dynamic response phenomena. Figure $9 \mathrm{a}, \mathrm{b}$ shows the phase portraits of the symmetric stoppers and asymmetric stopper, respectively. The velocity of the movable plate would jump up when came to an impact with a stopper every time. With an asymmetric stopper for each impact, the movable plate would experience attenuations of the displacement until the moment to the next impacts. We observed that the system is in periodic motion. However, due to the constraint from the limited space (top and bottom stoppers), the system is in asymmetric dynamics. We also observe that the system is still unstable status even with fierce impact from the stoppers. It concluded that the height of the asymmetric stopper can not only result in complicated dynamic motion of the movable plate but also modulated an RMS voltage shown in Figure 10.

\section{Conclusions}

In this study, we investigated a complicated dynamic response of the movable plate which came from strongly nonlinear asymmetric stoppers in MEMS variable dualcapacitor. We also found that the capacitance in the electrical circuit system related to the first-order derivative of the output voltage, and the variable dual-capacitor was strongly affected by the nonlinear electrostatic force. The good agreement results of the experiment and numerical 
(a)

$\underset{\text { Asymmetric stopper }}{\mathbf{A}} \underset{\text { symmetric stopper }}{\mathbf{B}}$

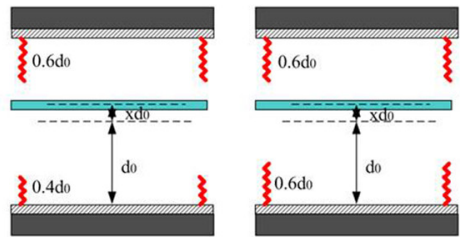

(c)

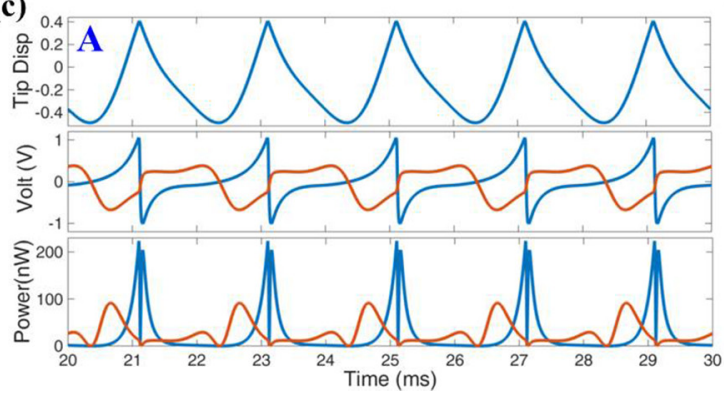

(b)
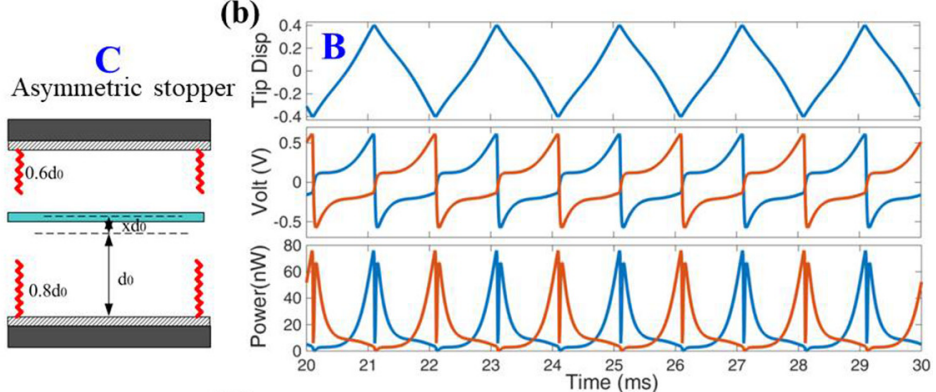

(d)

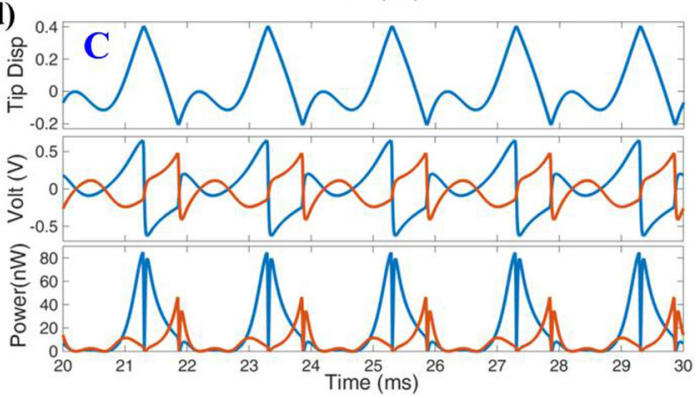

Fig. 8. Numerical simulation of the asymmetric stopper and symmetric stoppers with $500 \mathrm{~Hz}, 4 \mathrm{~g}$ excitation frequency, $0.6 \mathrm{~d}_{0}$ top stoppers: (a) the stoppers in dual-capacitor; (b) symmetry, $0.4 \mathrm{~d}_{0}$ bottom stoppers; (c) asymmetry, $0.6 \mathrm{~d}_{0}$ bottom stoppers; (d) asymmetry, $0.8 \mathrm{~d}_{0}$ bottom stoppers.
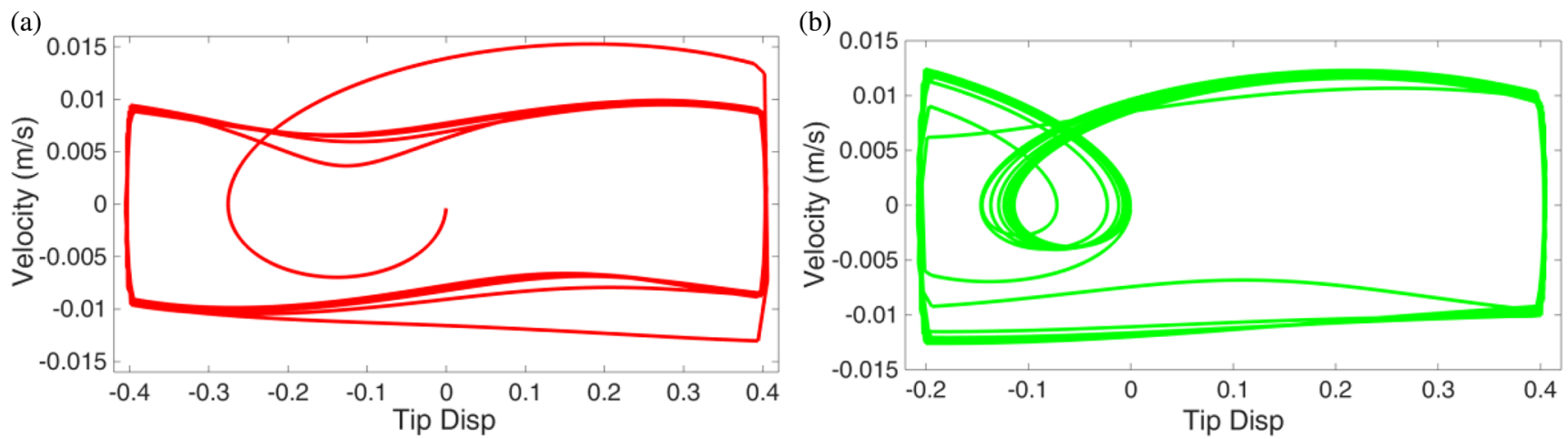

Fig. 9. Numerical simulation of the asymmetric stopper and symmetric stoppers with $500 \mathrm{~Hz}, 4 \mathrm{~g}$ excitation frequency, $0.6 \mathrm{~d}_{0}$ top stoppers: (a) phase portraits of symmetric stoppers; (b) phase portraits of the asymmetric stopper.

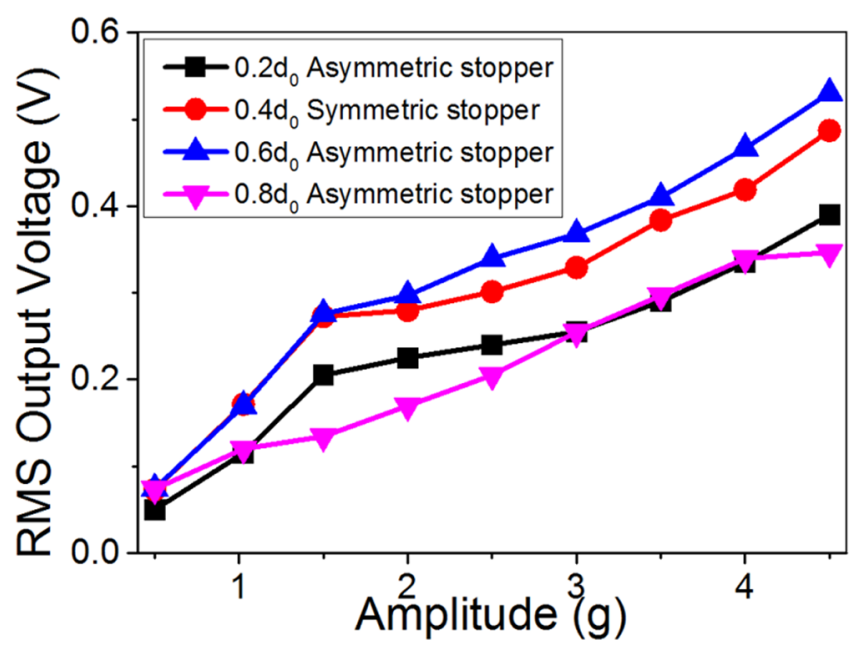

Fig. 10. RMS output voltage and dimensionless displacement of the movable of plate under external frequency $500 \mathrm{~Hz}, 16 \mathrm{~V}$ input voltage with different excitation amplitude and different length of bottom stoppers. 
simulation verified the feasibility of our analyzed mathematical model. Both the experimental and numerical simulation results showed that the forcing amplitude had not yet reached the maximum value, and no contact occurred between the movable plate and the fixed plate with $1.026 \mathrm{~g}$ amplitude. Furthermore, we demonstrated that the movable plate and stoppers had fierce impacts with phenomena of a softening effect of the system with $4 \mathrm{~g}$ amplitude. Moreover, the height and asymmetric structure of stoppers can not only result in a complicated dynamic response of the movable plate but also can modulate an RMS output voltage.

\section{Funding}

This study was supported by "the Fundamental Research Funds for the Central Universities" with No. 2242021R10020. This work was also sponsored by the National Science Foundation of China under Grant No. 61903081. The science and technology innovation Program of Hunan Province No. 2020RC2082.

Conflicts of interest. The authors declare no conflict of interest.

Jianxiong Zhu and Yunde Shi contributed the same to this work. The first author experimented and wrote the article. The second and the third author made important suggestions on the analysis of experimental data and made important improvements to this study.

\section{References}

[1] Y. Suzuki, Y. Miki, M. Edamoto, M. Honzumi, A MEMS electret generator with electrostatic levitation for vibrationdriven energy-harvesting applications, J. Micromech. Microeng. 20, 104002 (2010)

[2] J. Zhu, X. Liu, Q. Shi, T. He, Z. Sun, X. Guo, W. Liu, O. Sulaiman, B. Dong, C. Lee, Development trends and perspectives of future sensors and MEMS/NEMS, Micromachines 11, 541 (2020)

[3] J. Zhu, M. Zhu, Q. Shi, W. Feng, L. Long, B. Dong, A. Haroun, Y. Yang, P. Vachon, X. Guo, T. He, C. Lee, Progress in TENG technology - a journey from energy harvesting to nanoenergy and nanosystem (NENS), Ecomat. 2, 1-45 (2020)

[4] F. Shirazi, J. Velni, K. Grigoriadis, An LPV design approach for voltage control of an electrostatic MEMS actuator, J. Microelectrmech. Syst. 20, 302-311 (2011)

[5] J. Zhu, W. Song, R. Huang, Modulated pencil-drawn U-shaped piezoresistive graphite on compound fibers and its application for wind sensing, J. Electr. Mater. 47, 6518-6524 (2018)

[6] Y. Kim, S. Cho, H. Dong, J. Lee, K. Baek, Single chip dual plate capacitive proximity sensor with high noise immunity, IEEE Sensors J. 14, 309-310 (2014)

[7] G. Caruso, Broadband energy harvesting from vibrations using magnetic transduction, J. Vib. Acoust. Trans. ASME 137, 064503 (2015)
[8] K. Fan, J. Chang, F. Chao, W. Pedrycz, Design and development of a multipurpose piezoelectric energy harvester, Energy Convers. Manag. 96, 430-439 (2015)

[9] J. Zhu, C. Chen, X. Guo, Suspended polytetrafluoroethylene nanostructure electret film in dual variable cavities for selfpowered micro-shock sensing, Mater. Res. Express 6, 1-8 (2018)

[10] J. Zhu, X. Guo, D. Meng, M. Choi, I. Park, R. Huang, W. Song, A flexible comb electrode triboelectric-electret nanogenerator with separated microfibers for self-powered position, motion direction and acceleration tracking sensor, J. Mater. Chem. A 6, 16548-16555 (2018)

[11] J. Zhu, Y. Zhu, W. Song, H. Wang, M. Gao, M. Cho, I. Park, Zinc oxide enhanced piezoelectret polypropylene microfibers for mechanical energy harvesting, ACS Appl. Mater. Interfaces 10, 19940-19947 (2018)

[12] Z. Zeng, B. Ren, Q. Xu, D. Lin, W. Di, H. Luo, D. Wang, Excellent performances of energy Harvester using cantilever driving double-clamped $0.7 \mathrm{~Pb}(\mathrm{Mg} 1 / 3 \mathrm{Nb} 2 / 3) \mathrm{O}-3-0.3 \mathrm{PbTiO}$ (3) plates and symmetric middle-stops, Appl. Phys. Lett. 107, 173502 (2015)

[13] J. Zhu, W. Song, MEMS fabrication and frequency sweep for suspending beam and plate electrode in electrostatic capacitor, Solid-state Electr. 139, 94-100 (2018)

[14] J. Zhu, H. Zhu, Bulk silicon micromachined suspended fixedend $\mathrm{SiO}_{2}$ film capacitor for passive high-pass RC filter, Microsyst. Technolog. 23, 1-6 (2017)

[15] D. Galayko, A. Dudka, A. Karami, E. O'Riordan, E. Blokhina, O. Feely, P. Basset, Capacitive energy conversion with circuits implementing a rectangular charge-voltage cycle-part 1: analysis of the electrical domain, IEEE Trans. Circ. Syst. 62, 2652-2663 (2015)

[16] C. Chao, C. Chiu, C. Tsai, A novel method to predict the pull-in voltage in a closed form for micro-plates actuated by a distributed electrostatic force, J. Micromech. Microeng. 16, 986-998 (2006)

[17] B. Kim, H. Lee, Acoustic-thermal noise in a capacitive MEMS microphone, IEEE Sensors J. 15, 6853-6860 (2015)

[18] K. Yamamoto, T. Fujita, A. Badel, F. Formosa, K. Kanda, K. Maenaka, A design of mechanical frequency converter linear and non-linear spring combination for energy harvesting, PowerMEMS 557, 012065 (2014)

[19] S. Shaw, P. Holmes, A periodically forced piecewise linear oscillator, J. Sound Vibr. 90, 129-155 (1983)

[20] J. Qiu, Z. Feng, Parameter dependence of the impact dynamics of thin plates, Comput. Struct. 75, 491-506 (2000)

[21] A. Nayfeh, M. Younis, Dynamics of MEMS resonators under superharmonic and subharmonic excitations, J. Micromech. Microeng. 15, 1840-1847 (2005)

[22] J. Zhu, J. Lin, N. Yusek, M. Almasri, Z. Feng, Dynamic analysis and phenomena of MEMS capacitive power harvester subjected to low frequency excitation, Nonlinear Dyn. 79, 673-688 (2015)

[23] J. Zhu, N. Yuksek, M. Almasri, Z. Feng, Numerical modeling of dynamic response of miniature multi-impact electromagnetic device for low and wide range frequencies energy harvesting, Part C, J. Mech. Eng. Sci. 233, 2400-2409 (2019)

[24] J. Lin, J. Zhu, M. Sonje, Y. Chang, Z. Feng, M. Almasri, Two-cavity MEMS variable capacitor for power harvesting, J. Micromech. Microeng. 22, 6 (2012) 
[25] E. Ferro, P. López, V. Brea, D. Cabello, Dynamic joint model of capacitive charge pumps and on-chip photovoltaic cells for CMOS micro-energy harvesting, Int. J. Circ. Theor. Appl. 44, 1874-1894 (2016)

[26] N. Ramli, T. Arslan, N. Haridas, W. Zhou, Design, simulation and analysis of a digital RF MEMS varactor using thick SU-8 polymer, Microsyst Technol. 24, 473-482 (2018)

[27] H. Nieminen, V. Ermolov, K. Nybergh, S. Silanto, T. Ryhanen, Microelectromechanical capacitors for RF applications, J. Micromech. Microeng. 12, 177-186 (2002)

[28] M. Toki, Y. Tsuzuki, T. Mitsuoka, Measuring method of active impedance of crystal oscillator circuits, Electr. Commun. Jpn. (Part II: Electronics) 68, 68-76 (1985)

[29] B. Dong, Y. Ma, Z. Ren, C. Lee, Recent progress in nanoplasmonics based integrated optical micro/nano-systems, J. Phys. D 53, 213001 (2020)

[30] Z. Ren, Y. Chang, Y. Ma, K. Shih, B. Dong, C. Lee, Leveraging of MEMS technologies for optical metamaterials applications, Adv. Opt. Mater. 8, 1900653 (2020)

[31] H. Prashad, Theoretical evaluation of impedance, capacitance and charge and accumulation on roller bearings operated under electrical fields, Wear 125, 223-239 (1988)
[32] E. Gallagher, W. Moussa, A study of the effect of the fringe fields on the electrostatic force in vertical comb drives, Sensors 14, 20149-20164 (2014)

[33] S. Banerjee, M. Levy, M. Davis, B. Wilkerson, Exact and approximate capacitance and force expressions for the electrostatic interaction between two equal-sized charged conducting spheres, IEEE Trans. Ind. Appl. 53, 2455-2460 (2017)

[34] C. Je, J. Jeon, S. Lee, W. Yang, MEMS capacitive microphone with dual-anchored membrane, Proceedings 1, 342 (2017)

[35] J. Dennis, A. Ahmed, M. Khir, A. Rabih, Modelling and simulation of the effect of air damping on the frequency and quality factor of a CMOS-MEMS resonator, Appl. Math. Inf. Sci. 9, 729-737 (2015)

[36] J. Zhu, X. Guo, R. Huang, Asymmetric disappearance and periodic asymmetric phenomena of rocking dynamics in micro dual-capacitive energy harvester. 2018 International Symposium on Aerospace, Automotive, Mechanical and Materials Engineering 1971, 040002 (2018)

[37] J. Lin, J. Zhu, Y. Chang, Z. Feng, M. Almasri, Surface micromachined MEMS capacitors with dual-cavity for energy harvesting, J. Microelectromech. Syst. 22, 1458-1469 (2013)

Cite this article as: J. Zhu, Y. Shi, F. Ma, Symmetry and asymmetry from MEMS variable capacitor by nonlinear micro stoppers, Mechanics \& Industry 22, 41 (2021) 